\title{
Supervisory Adaptive Control for the Structural Vibration of a Coordinate-Measuring Machine
}

\author{
Jianjun Shi* and Jun $\mathrm{Ni} \dagger$ \\ *Department of Industrial and Operations Engineering; †Department of Mechanical Engineering and Applied Mechanics, \\ The University of Michigan, Ann Arbor, USA
}

\begin{abstract}
A supervisory adaptive control approach has been developed for a structural vibration control of a coordinate measuring machine, which is a dynamic system with time varying system model order and parameters. An on-line dynamic data system modelling algorithm is used to identify the system model order and parameters simultaneously. Based on the identified model, a predictive control algorithm is applied to generate control commands. A supervisory strategy with several monitoring indices and decision-making rules is proposed to supervise the modelling and control processes. The developed supervisory adaptive control approach has been implemented in a digital signal processor board for structural vibration control. Experimental results indicate a $75 \%$ reduction in the peak-to-peak vibration and an $80 \%$ reduction in the settling time.
\end{abstract}

Keywords: Coordinate measuring machine; Model order identification; On-line modelling; Supervisory adaptive control; Vibration control

\section{Introduction}

The coordinate measuring machine (CMM) has been widely used in manufacturing as a precision measurement gauge. However, the performance of CMMs is severely limited by dynamic deflection of the measurement problem which persists for a period of time after motion is completed. This problem becomes more dominant for a high-speed CMM, where a lightweight manipulator is generally used to reduce the driving torque requirements and to enable the CMM arm to respond faster. The lighter mechanisms are more likely to deform elastically, causing a higher level of vibration during movement. The settling time required for the vibration to decay delays subsequent operations, and conflicts with the demand for increased CMM measurement speed. In addition, the vibration

Correspondence and offprint requests to: Dr Jianjun Shi, Department of Mechanical Engineering and Applied Mechanics, The University of Michigan, 3424 GG Brown, Ann Arbor, Michigan 48109-2125, USA. of the CMM manipulator also causes a dynamic measurement error, which limits the precision of the CMM. As a result, reducing the vibration of the CMM manipulator to achieve high measurement speed and to maintain high measurement precision is a challenging research problem.

Lu et al. [1] studied CMM vibration control problems using an integrated lattice filter adaptive control system. In this approach, a lattice filter was applied to identify the vibration system model. A minimum variance (MV) control algorithm was linked with the identification algorithm. Simulation and experiments indicated an $80 \%$ reduction in the system settling time. However, owing to the limitations of the minimum variance control, the control performance was very sensitive to system time delays and variations. In addition, no supervision was designed for the adaptive controller. Thus, "the belts were tensioned and air bearings were adjusted to their maximum stiffness" to obtain a fixed time delay. This is not the normal CMM working condition. As a result, further study on the vibration control of a CMM is important. One direction of the study is to design a supervisory adaptive controller based on a more effective on-line modelling algorithm and predictive control strategy.

Adding a supervisory strategy is very important in an adaptive control. An adaptive control method is often applied to a system with time-varying parameters and structure. The adoption of the real-valued function has led to a sophisticated and comprehensive mathematical theory of feedback control. Differential or difference equations are used to model continuous-time and discrete-time dynamic systems respectively, and powerful synthesis methods have been derived [2]. These adaptive strategies attempt to identify (modify) the model in real-time and to update the control law continuously. However, both modelling and adaptation algorithms require a priori assumptions. Once these assumptions are made, they are often neglected during the design and subsequent verification phases. This can result in the actual system behaviour being significantly different from the designed, or desired behaviour, and even failing to satisfy the prescribed specifications. Efforts have been made to develop knowledgebased adaptive control or supervisory control strategies. The first steps in this direction were proposed in [3-6]. Almost 
all of them concentrated on the "bursting problems" in parameter estimation. Several supervisory functions for the adaptive loop were proposed in [7]. Isermann and Lachmann [8] proposed a supervision level, which tries to monitor faulty functions as early as possible and to take appropriate actions in order to guarantee a satisfactory behaviour of the adaptive control loops.

In general, most of the research efforts in this area are focused on the development of the concepts of supervisory adaptive control. Little literature could be found in supervisory adaptive control considering model order identification. Furthermore, few applications of the supervisory adaptive control techniques have been reported to date.

In this paper, a supervisory adaptive control approach is proposed using an on-line dynamic data system (DDS) modelling developed by Shi [9] and predictive control techniques from Bitmead et al. [10]. The on-line modelling algorithm is used to identify the system model order and parameters simultaneously. A predictive control algorithm is implemented to generate control actions based on this identified model. All these modelling and control processes are supervised by the developed supervisory strategy. According to the principle of "higher intelligence, lower precision" [11], the approach consists of three levels (see Fig. 1). The highest level is a supervision level to supervise the other two levels. The middle level is to finalise controller parameter adjustment and system identification. The lowest level is a predictive controller, which is used to control the process directly. The supervisory adaptive control approach developed has been successfully implemented in a digital signal processor board (DSP), and applied to a structural vibration control problem for a coordinate measuring machine (CMM).

This paper is organised as follows. Following a brief introduction, the on-line system modelling algorithm, the predictive control algorithm and the supervisory strategy are presented in Section 2. Section 3 outlines the implementation on the CMM structural vibration control and its experimental results are given. The conclusions are summarised in Section 4.

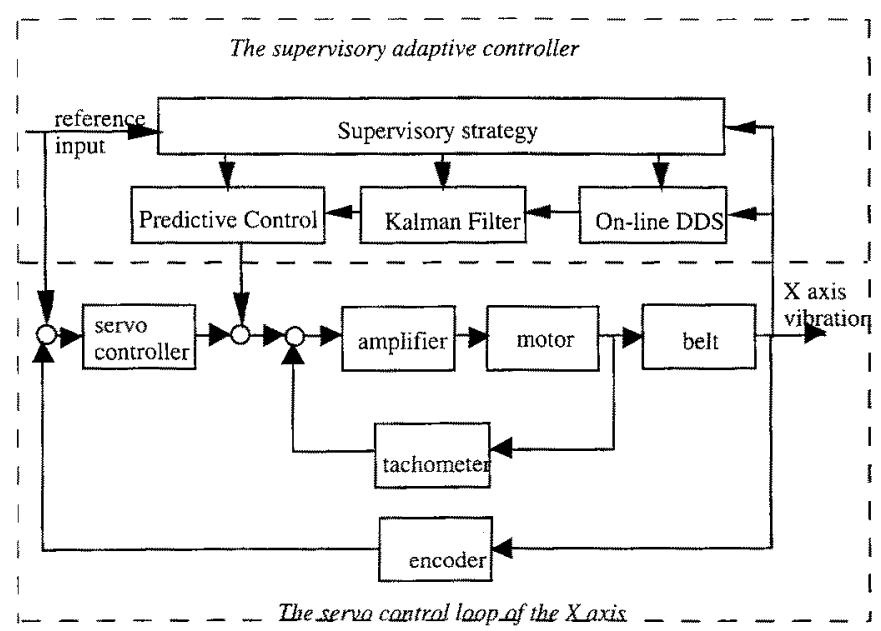

Fig. 1. The supervisory adaptive control for a CMM structural vibration control.

\section{Supervisory Adaptive Control Algorithm}

\subsection{On-line System Identification}

Consider a single-input single-output $\operatorname{ARX}(n, m)$ process,

$$
\left(1+\sum_{i=1}^{n} a_{i} z^{-i}\right) y(t)=\left(\sum_{i=0}^{m} b_{i} z^{-i}\right) z^{-1} u(t)+v(t)
$$

There are two basic tasks in the system identification process, which are:

1. Model order determination

2. Model parameter estimation.

To identify the model order and parameters simultaneously, a candidate model set is defined as:

$$
C_{\mathrm{ARX}}=\left\{\operatorname{ARX}(n, n-1) \mid n=1,2, \ldots, n_{\max }\right\}
$$

where $n_{\max }$ is the highest model order. After defining (2), the system model order and parameters can be identified using a model information matrix, $\mathbf{M}(t)$, and its factorization [9].

First define a model information matrix as shown in Appendix A. The matrix can be decomposed into its UDU ${ }^{\mathrm{T}}$ form [9]:

$$
\mathbf{M}(t)=\mathbf{U}(t) \mathbf{D}(t) \mathbf{U}^{T}(t)
$$

where $\mathrm{U}(t)$ and $\mathbf{D}(t)$ have the form

$$
\left.\begin{array}{ccccc}
\mathrm{U}(t)= & & & & \\
1-\theta_{0}^{*}(\mathrm{t}) & & & & \\
1 & -\theta_{1}(\mathrm{t}) & & & \\
& 1 & -\theta_{1}^{*}(\mathrm{t}) & & \\
& & 1 & -\theta_{n-1}^{*}(\mathrm{t}) & \\
& & & 1 & -\theta_{n}(\mathrm{t}) \\
& & & & 1
\end{array}\right]
$$

and

$$
\begin{aligned}
& \mathbf{D}(t)=\operatorname{diag}\left(J_{0}^{-1}(t), J_{0}^{*-1}(t), J_{1}^{-1}(t),\right. \\
& \left.\ldots, J_{n}^{-1}(t), J_{n-1}^{*-1}(t), J_{n}^{-1}(t)\right)
\end{aligned}
$$

The properties of matrices $\mathbf{U}(t)$ and $\mathbf{D}(t)$ are explained in the following remarks. The proof can be found in [9].

Remark 1: $\mathbf{U}(t)$ is an upper triangular $(2 n+1) \times(2 n+1)$ matrix with ones on the principal diagonal. The vector $\theta_{k}(t)$ $(k=1,2, \ldots, n)$ is the weighted least-squares estimate of the parameters of a $k$ th-order model where the parameter vector has the form

$$
\begin{aligned}
\theta_{i k}(t)= & {\left[-a_{k}(t-n+k), b_{k-1}(t-n+k),\right.} \\
& -a_{k-1}(t-n+k), \\
\ldots,- & \left.a_{1}(t-n+k), b_{0}(t-n+k)\right]^{T}
\end{aligned}
$$

Thus, all parameter estimates of the candidate model set (2) are obtained simultaneously from $\mathbf{U}(t)$. Note that a forgetting factor is used in the $\mathbf{M}(t)$ (Appendix A) and the parameter estimates are the least-squares estimates with discounted 
measurements. This makes it feasible to track a time-varying process.

Remark 2. $\mathbf{D}(t)$ is a $(2 n+1) \times(2 n+1)$ diagonal matrix. In the diagonal components, $J_{k}(t),(k=1,2, \ldots, n)$ is the criterion that is minimised to determine the weighted leastsquares estimates for the $k$ th-order model. In other words, $J_{k}(t)$ (see Appendix for definition) is the weighted sum of the squares of the residuals for a $k$ th-order model at time $t$. If no forgetting factor is applied in the algorithm, $J_{k}(t)$ is the unweighted sum of the squares of the residuals (RSS) for the $k$ th-order model. Thus, the information contained in the matrix $\mathbf{D}(t)$ can be used in the order determination.

Remark 3. In the $\mathbf{U}(t)$ and $\mathbf{D}(t)$ matrices, $J_{k}^{*}(t)$ and $\theta_{k}^{*}(t)$ $(k=1,2, \ldots, n-1)$ are obtained as part of the $\mathbf{U D U}^{\mathbf{r}}$ factorisation form of the $\mathbf{M}(\mathbf{t})$. They are intermediate variables and have no physical significance.

In practice, a recursive algorithm is used to get the $\mathbf{U D U}^{\mathbf{T}}$ factorisation form of the $\mathbf{M}(t)$. The recursive algorithm, including the model order determination algorithm, is summarised in Table 1. The notations and explanations for Table 1 are given in Remark 4.

\section{Remark 4.}

1. $D_{i j}(t)$ and $U_{i j}(t)$ are the components of matrix $\mathbf{D}(t)$ and $\mathbf{U}(t)$, respectively.

2. $R_{i}(t)$ is the sum of the squares of the residuals of the $i$ th model at time $t$; and $R_{i}(t, N)$ is the sum of the squares of the residuals of the $i$ th model at time $t$ over time interval $[t-N, t]$.

3. $F_{i, j}$ is the statistic of the $i$ th- and $j$ th-order model and will be used in the $F$-test for the order determination.

4. $\lambda(t)$ in step 4 is a forgetting factor; $N$ in steps 8 and 9 is the window width designed for order determination of a time varying process.

5. $\epsilon_{j}(t)$ is the residual of the $j$ th-order model at time $t$.

6. All other variables are intermediate variables or vectors.

\subsection{Predictive Control}

A predictive control algorithm is used in the proposed supervisory adaptive control. The predictive control algorithm consists of three steps at each sampling instant. First, the effects of the control variables on the output are predicted. Secondly, the control action is determined by minimising a receding quadratic cost function of the predicted output errors and the control steps. Finally, the resulting control action is applied to the system. The above three steps are repeated at every sampling interval. A complete recent survey of predictive control is given in [10].

For simplicity of derivation, the ARX model is transformed into state space form:

$$
\begin{aligned}
X(k+1) & =A X(k)+B U(k)+W(k) \\
Y(k) & =C X(k)+V(k)
\end{aligned}
$$

Table 1. The procedure for the recursive on-line system modelling.

Step 1: construct $\Phi(t)=[y(t-n), u(t-n), \ldots, y(t-1)$,

$$
u(t-1), y(t)]^{r}
$$

Step 2: calculate $\mathbf{f}(t)=\mathbf{U}^{T}(t-1) \Phi(t), \quad \mathbf{g}(t)=\mathbf{D}(t-1) \mathbf{f}(t)$

$$
\text { and } \quad \mathbf{U}(0)=0 ; \mathbf{D}(0)=\rho \mathbf{I}(\rho>0 \text {, is a large number }) \text {. }
$$

Step 3; let $\quad \alpha_{0}(t)=\lambda(t)$ for $j=1,2, \ldots, 2 n_{\max }+1$, repeat steps 4 to 6 ;

Step 4: calculate $\alpha_{j}(t)=\alpha_{j-1}(t)+\mathbf{f}_{j}(t) \mathbf{g}_{j}(t)$,

$$
\mathbf{D}_{j j}(t)=\frac{\alpha_{j-1}(t) \mathbf{D}_{j i}(t-1)}{\alpha_{j}(t) \lambda(t)}
$$

and let $\quad \mathbf{s}_{j}(t)=\mathbf{g}_{j}(t), \quad \epsilon_{j}(t)=-\frac{\mathbf{f}_{j}(t)}{\alpha_{j-1}(t)}$

Step 5: for $\quad i=1,2, \ldots, j-1$, repeat step 6 ;

Step 6: calculate $\mathbf{U}_{i j}(t)=\mathbf{U}_{i j}(t-1)+\mathbf{s}_{i}(t) \epsilon_{j}(t)$; $\mathbf{s}_{i}(t)=\mathbf{s}_{i}(t-1)+\mathbf{U}_{i j}(t) \mathbf{s}_{j}(t)$.

Step 7: $\quad$ calculate $R_{i}(t)=R_{i}(t-1)+\lambda(t)\left(\frac{\alpha_{i}(t)}{\alpha_{i-1}(t)}-1\right) J_{i}(t-1)$ with initial condition $R_{i}(0)=J_{i}(0)$.

Step 8: calculate:

$$
\begin{array}{ll}
R_{i}(t, N)=R_{i}(t)-R_{i}(t-N) & (\text { when } t>N), \\
R_{i}(t, N)=R_{i}(t) & \text { (when } t \leq N)
\end{array}
$$

Step 9: calculate $F_{i j}(t)=$

$$
\begin{aligned}
& \frac{R_{i}(t-i, N)-R_{j}(t-j, N)}{2(i-j)} / \frac{R_{j}(t-j, N)}{\operatorname{Min}(t-j, N)-2 j} \\
& \sim F(2(i-j), \operatorname{Min}(t-j, N)-2 j)
\end{aligned}
$$

where $i, j$ are the model orders, and

$$
\operatorname{Min}(t, N)=\left\{\begin{array}{cc}
N, & t>N \\
t, & t \leq N
\end{array} .\right.
$$

where $X(k) \in \mathbf{R}^{n}, Y(k) \in \mathbf{R}^{1}, W(k) \in \mathbf{R}^{n}, V(k) \in \mathbf{R}^{1}$ and $U(k) \in \mathbf{R}^{1}$.

Assuming $U(k+i)=0,\left(i=1,2, \ldots, N_{p}\right)$, the $i$-step ahead prediction of the output $\hat{Y}(k+i \mid k)$ can be obtained from its expected value, as

$$
\begin{aligned}
& \hat{X}(k+i \mid k)=A^{i} \hat{X}(k)+A^{i-1} B U(k) \\
& \hat{Y}(k+i \mid k)=C A^{i} \hat{X}(k)+C A^{i-1} B U(k)
\end{aligned}
$$

where $\hat{X}(k)$ is the state estimation from a Kalman filter.

To get a predictive control law, consider the following receding control index:

$$
J(k)=\frac{1}{2} E\left\{\sum_{i=1}^{N_{p}}\left(\hat{Y}^{2}(k+i \mid k)+R U^{2}(k)\right)\right\}
$$

where $N_{p}$ is the prediction horizon; $R$ is the weighting coefficient and $R>0$.

By minimising the control index (9), i.e. setting $\mathrm{d} /(k) /$ $\mathrm{d} U(k)=0$, the predictive control law is obtained as: 


$$
\begin{aligned}
& U(k)=-\left\{\sum_{i=1}^{N_{p}}\left(C A^{i-1} B\right)^{T}\left(C A^{i-1} B\right)+R\right\}^{-1} \\
& \left\{\sum_{i=1}^{N_{p}}\left(C A^{i-1} B\right)^{T}\left(C A^{i} \hat{X}(k)\right)\right\}
\end{aligned}
$$

\subsection{Supervisory Strategy}

\section{Order Determination by F-test}

In the proposed on-line modelling approach, the system model order is determined by checking the significance level of model residual error decrease. The $F$-test is used in the order determination. However, the $F$-test is a statistical test with a probability of having errors greater than zero. As a result, some incorrect results may occur from a statistical point of view. This problem can be avoided in the order determination strategy by adding the supervisory rule: a newly determined model order is acceptable if, and only if, the number of consecutive $F$-tests consistently indicate an order change that is greater than a pre-set threshold $N_{f}$.

\section{Supervision of the Measurement Noise Mean}

In the on-line modelling, the measurement mean is assumed to be zero. If the noise means is not zero, the estimate will have a bias which could lead to unstable performance. To get a reliable estimation result, an estimate of the mean value is used in the on-line modelling algorithm. The estimate of the observation mean at the $k$ th instant is

$$
M(k)=\frac{k-1}{k} M(k-1)+\frac{1}{k} y(k)
$$

\section{Detection of Identifiability Conditions}

In a closed-loop system, the variation of the process input and output signal can become rather small when the controller works well and when no external disturbances are exciting the control loop. Thus, no dynamic information about the process can be gained from the measured input and output signals. In many cases, this will result in the "bursting" problems in parameter estimation [12]. This situation is usually indicated by an increasing variance of parameter estimates which drifts to wrong values.

To overcome this problem, a simple remedy is to automatically switch off the parameter estimation if the identifiability condition is violated. The identifiability conditions can be detected by checking the model information matrix $\mathbf{M}(t)$ (Appendix A).

If the $\mathbf{M}(t)$ matrix is not positive definite, the identification problem may become unsolvable. The parameter estimation process should be stopped immediately and continued only when the system is identifiable again.

\section{Detection of Abrupt System Dynamic Change and Reaction}

In an adaptive control system, an abrupt change in the system dynamics may lead to unstable on-line modelling or control performance. Unexpected and abrupt changes in system dynamics should be detected and responded to as soon as they occur. Different algorithms and approaches have been developed [13]. In this paper, a recursive $F$-test and cumulativesum (CUSUM) control chart detect approach is proposed using the residual sequence obtained from the on-line modelling algorithm. To simplify the abrupt change detection algorithm, only the residual from the model with the highest model order in the candidate model set is used in the test. The residual is represented as $\epsilon(t)$, and $\epsilon(t)=\epsilon_{n \max }(t)$.

Recursive F-test. The $F$-test is used to detect variance shift in the residual sequence process. Two sliding windows are used consequently to calculate the standard deviation of residuals. Thus, we have

$$
\mu_{1}=\frac{1}{N_{1}+1} \sum_{j=k-N_{1}-N_{2}-1}^{k-N_{2}-1} \epsilon(j), \quad \mu_{2}=\frac{1}{N_{2}+1} \sum_{j=k-N_{2}}^{k} \epsilon(j)
$$

and

$$
\begin{aligned}
& S_{1}^{2}=\frac{1}{N_{1}} \sum_{j=k-N_{2}-N_{1}-1}^{k-N_{2}-1}\left[\epsilon(j)-\mu_{1}\right]^{2} \\
& S_{2}^{2}=\frac{1}{N_{2}} \sum_{j=k-N_{2}}^{k}\left[\epsilon(j)-\mu_{2}\right]^{2}
\end{aligned}
$$

The statistic, $f=S_{2}^{2} / S_{1}^{2}$, follows $F$ distribution with $\left(N_{2}, N_{1}\right)$ degrees of freedom. By specifying a confidence level $\alpha$, the following rules apply:

$$
\begin{aligned}
& H_{0}: \text { no significant change in standard deviation (no } \\
& \text { jump); } \\
& H_{1}: \text { with a significant change in standard deviation } \\
& \text { (jump); } \\
& \text { If } f<F_{\mathrm{\alpha}} \text {, accept } H_{0} \text {; otherwise, reject } H_{0} \text { and accept } \\
& H_{1} \text {. }
\end{aligned}
$$

where $F_{\alpha}$ is obtained from an $F$ distribution table.

The Cumulative-Sum (CUSUM) Chart. The CUSUM chart is used to evaluate the hypothesis that if the process has remained stable, the true mean of the residuals should be zero. If the hypothesis is contradicted by the data, there is a significant reason to believe that a change has occurred which has affected the mean of the residuals. If the hypothesis cannot be disproved by the data, it is assumed that no fault has occurred which would affect the mean of the residuals.

The CUSUM chart has been introduced in many quality control books. The calculation of the positive and negative CUSUMs follows [14]:

$$
\begin{aligned}
& S_{H}(i)=\max \left[0, \epsilon(i)-b+S_{Y I}(i-1)\right] \\
& S_{L}(i)=\max \left[0,-\epsilon(i)-b+S_{L}(i-1)\right]
\end{aligned}
$$

where $b=\Delta / 2, \Delta$ is the shift in the mean of residuals which is being monitored.

A sporadic fault is indicated at time $k$ if $S_{H}(i)>h$ or $S_{L}(i)<h$, where $h$ and $\Delta$ are the parameters of CUSUM chart. In general, the values of $\Delta$ and $h$ determine test sensitivity. 


\section{Supervision of the Adaptive Control While the On-line Modelling is in the Transient Period}

In the adaptive control, an on-line modelling method is used for the system control loop. However, there is always a transient period required for the on-line modelling technique to converge to an adequate model. During this transient period, the identified model is generally inadequate. If the transient model is used directly in adaptive control, a poor, or even unstable, control performance may result.

The supervision of the adaptive control during the transient period of the on-line modelling is application dependent. Some alternative control approach, such as PID controller, fuzzy controller, expert controller and dual controller, etc., can be applied. In this paper, a supervisory control rule is proposed for CMM vibration control and is presented in the next section.

\section{Implementation on the CMM Structural Vibration Control}

\subsection{Experimental Set-up}

The structure of the CMM used in the experiments is shown in Fig. 2. The CMM is a three axis (referred to as the $X, Y$ and $Z$ axes) horizontal arm CMM driven by three d.c. servo motors. In this paper, the symbols, $X_{\max }, Y_{\max }$, and $Z_{\max }$, are used to indicate the maximum travel limit of the $X-, Y$-, and $Z$-axes, respectively. The $X$-axis is selected to demonstrate the effectiveness of the developed supervisory adaptive control approach.

The servo system and vibration control loop for the $X$-axis is shown in Fig. 1. An EG\&G d.c. servo motor drives the carrier along the $X$-axis. A tachometer and an encoder are used for speed and position feedback. A servo system

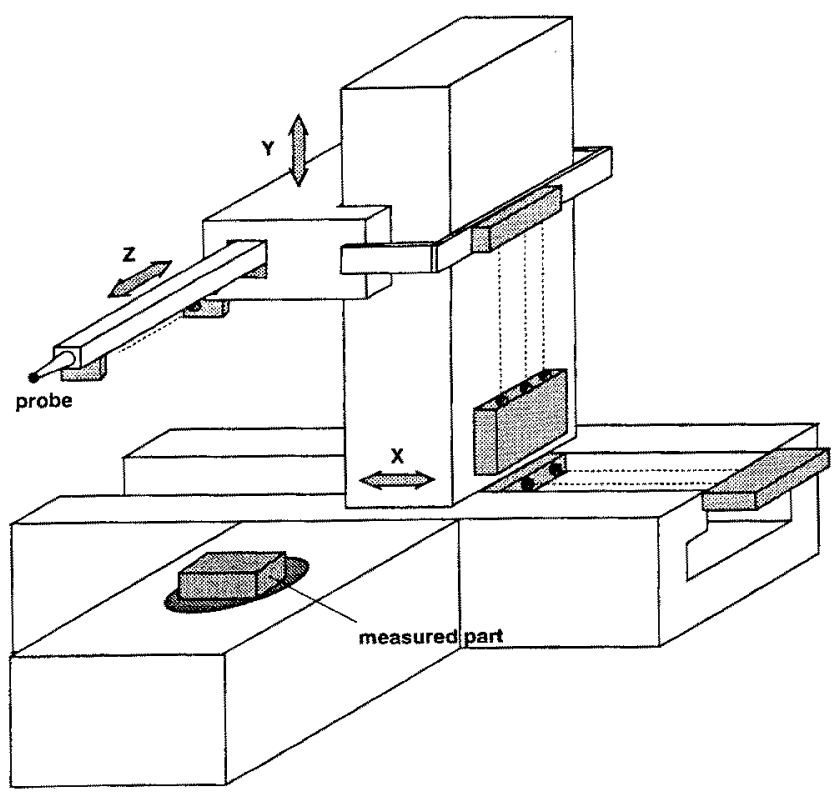

Fig. 2. The coordinate measuring machine (CMM). controller controls both speed and position along the $X$ axis. The vibration signal is measured using a PCB 308A2 accelerometer (1000 mv/g high sensitivity) and a Kistler 5004 dual mode amplifier. A step reference control signal provides the input to the CMM controller. The active vibration control signal, generated by a digital signal processor (DSP) board (TMS320C30) integrated with a Dell $386 \mathrm{PC}$ computer, is added to the reference input signal to suppress the vibration of the CMM horizontal arm in the direction of the $X$-axis.

The vibration signals are prefiltered by a low-pass filter with a cut-off frequency of $50 \mathrm{~Hz}$ before reaching the DSP board. An A/D channel of the DSP board is used to sample the sensor signal. The DSP processor computes the supervisory adaptive control command signal and sends it through the $D$ / A output channel of the DSP board. The command signal is sent directly to the $E G \& G$ velocity servo controller which drives the d.c. motor.

In the experiment, the position control loop was not included.

\subsection{Special Sueprvisory Concerns in the CMM Experiments}

\section{Characteristics of CMM Horizontal Arm Vibration}

The vibration of the horizontal arm of the CMM has three essential variational characteristics: length of arm dependency, direction of $x$-motion dependency, and a time variational dependency.

First, consider the length of arm dependency. From the structure of the CMM, it is obvious that the CMM will have different vibration modes with the horizontal at different extensions. For example, the vibration characteristics with the horizontal arm fully extended will be very different from those with the horizontal arm fully retracted. Figure 3 shows the vibration spectrum of the horizontal arm in the extended and retracted positions. It is clear that the vibration spectrum at the fully extended position has more peaks than at the fully retracted position: there are noo peaks at $21.2 \mathrm{~Hz}$, $26.5 \mathrm{~Hz}$ and $34.2 \mathrm{~Hz}$ at the fully retracted position.

Another important property is the direction of motion in $X$-direction. Figure 4 shows the difference in vibration spectra when the CMM is moving in the positiive and negative

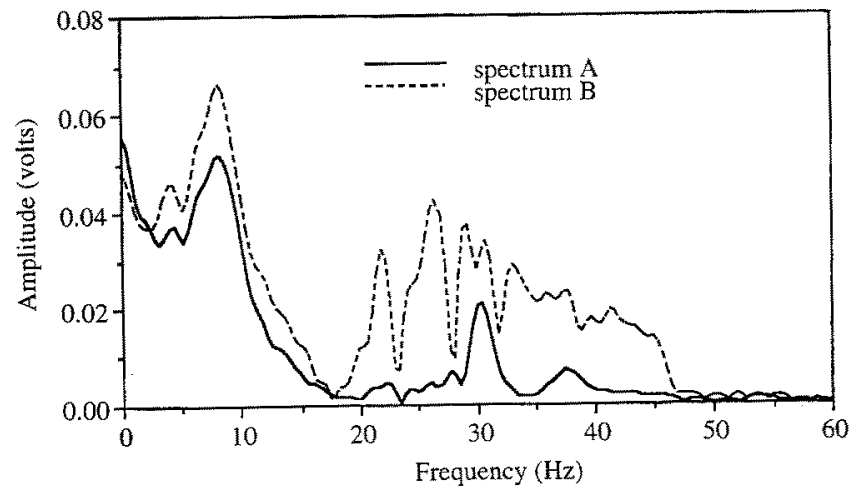

Fig. 3. Position dependent characteristics. A at position $(0,0,0)$; B at position $\left.(0),, Z_{\text {max }}\right)$. 


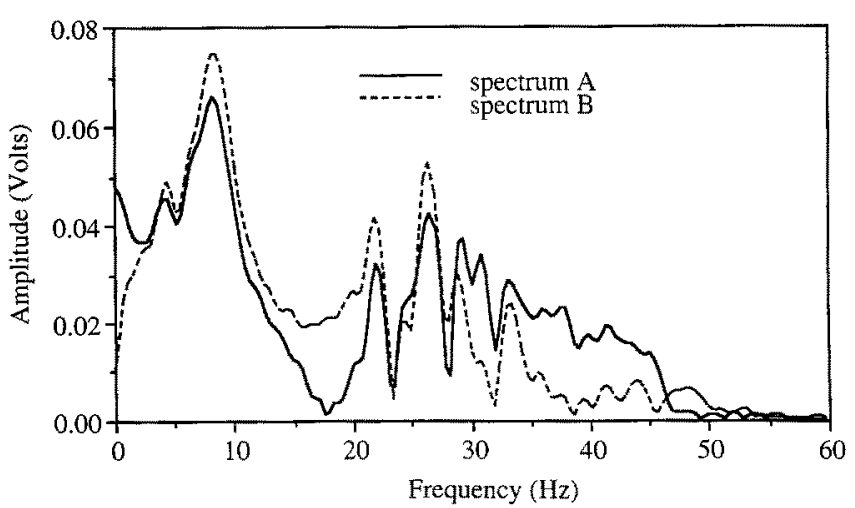

Fig. 4. Direction dependent characteristics at position $\left(0,0, Z_{\max }\right)$. A: movement in $+X$ direction; $\mathrm{B}$ : movement in $-X$ direction).

directions along the $X$-axis. One example is the peak at $30.8 \mathrm{~Hz}$. This peak is absent when the arm is moving in the negative $X$-direction.

The time-varying property of the CMM vibration is due to the movement of the horizontal arm during measurement. The direction-dependent property and the position-dependent property will be reflected as time-varying properties. However, if an on-line modelling technique is applied to the system input and output, a time-varying model can be obtained.

These characteristics indicate that the $\mathrm{CMM}$ vibration is a dynamic system with time-varying model order and parameters. Thus, when the adaptive control scheme is used, both the model order and parameters should be identified simultaneously. Also, since the CMM vibration model is direction dependent, the system model undergoes a sudden change when the direction of travel is reversed. Thus, the on-line modelling passes through a transient period at each direction change. This will pose a greater challenge to the adaptive control problem.

\section{Control Strategy During the Transient Modelling Periods}

The concept of the supervisory strategy is to use a priori knowledge of the CMM horizontal arm vibration system. When the arm motion is reversed, an abrupt change occurs. In this event, the on-line modelling algorithm needs a transient time to converge to the system model. Thus, the supervisory strategy will be applied to the system first. After the transient modelling period has passed, the adaptive control is used to control the vibration directly.

In this application, the prior knowledge used is that the vibration control can be reduced by smoothing the reference input. However, smoothing the reference input signal will also reduce the CMM throughput. A trade-off between vibration and through-put must be achieved. In the experiment, the supervisory control input is designed as:

$$
u_{s}(k)=u_{s}(k-1)+a_{s}\left(u_{\mathrm{ref}}(k)-u_{s}(k-1)\right)
$$

where $u_{\text {ref }}$ is the reference input; $u_{s}$ is the input after the supervisory rule is applied to the system; $a_{s}\left(0<a_{s} \leq 1\right)$ is the trade-off coefficient to balance the vibration and the throughput. If $a_{s}$ is small, a small vibration signal can be achieved. However, the CMM throughput will be lowered owing to the smaller reference control signal. If $a_{s}$ is large, the CMM may have higher throughput, but the vibration signal will be larger.

\section{Supervision of the Kalman Filter for the State Estimation when the Vibration Model Switches}

If an abrupt change occurs, the Kalman filter used in the state estimation will also have a transient time. However, since the Kalman filter gain converges to its static value (generally it is a small value), it would be difficult for the Kalman filter to track the state variable after the model switches. To improve the tracking ability of the Kalman filter when the model switches, the state covariance matrix $P(t)$ has to be reset. Thus, the following strategy is used:

IF $\{$ An abrupt process dynamic change is detected THEN $\left\{\right.$ Let $\left.P(s)=P_{s}^{*} I\right\} . P_{s}$ is a predefined positive number which can cover the maximum parameter change during the model switches; $I$ is the identity matrix.

\subsection{Experimental Results}

The supervisory adaptive control algorithm is programmed using the SPOX C language, combined with assembly language, and implemented in a digital signal processor (TMS320C30). In the experiments, the following parameters are selected:

1. Data acquisition: sampling frequency, $400 \mathrm{~Hz}$; low-pass filter, $50 \mathrm{~Hz}$.

2. On-line modelling: maximum order of the candidate model set: $n=8$; the initial model order: 2 ; initial coefficient values: zero; forgetting factor: 0.99 ; receding window length used in the on-line order determination: 120; the confidence level used in the on-line order determination: 0.99 .

3. Predictive control: predictive horizon: $N_{p}=4$; weighting coefficients $R=0.05$.

4. Supervisory strategy: order determination $N_{f}=3$; abrupt change detection $N_{1}=N_{2}=50, F_{\alpha}=2.73$ and $\Delta=0.1$; $a_{s}=0.95$ in the supervisory control input equation (15).

\section{On-line Modelling Experimental Results}

For supervisory adaptive control purposes, the input signal given by equation (15) is used to drive the CMM. The output signal is measured with an accelerometer. Both the input and output signals are used for the on-line modelling. Equation (2) is selected as the candidate model set in the experiment. No further assumptions are made about the system model order or parameters.

The on-line DDS modelling experiment was performed with the horizontal arm at position $\left(0,0, Z_{\max }\right)$. The on-line order determination result is shown in Fig. 5. Figure 6 shows the two-step-ahead output prediction, based on the identified model, compared with the real output. From the figure, we see that the identified model is adequate. It should be emphasised that the model used in Fig. 6 was obtained using the on-line DDS method without any assumptions on, or knowledge of, the vibration system model order and parameters. 


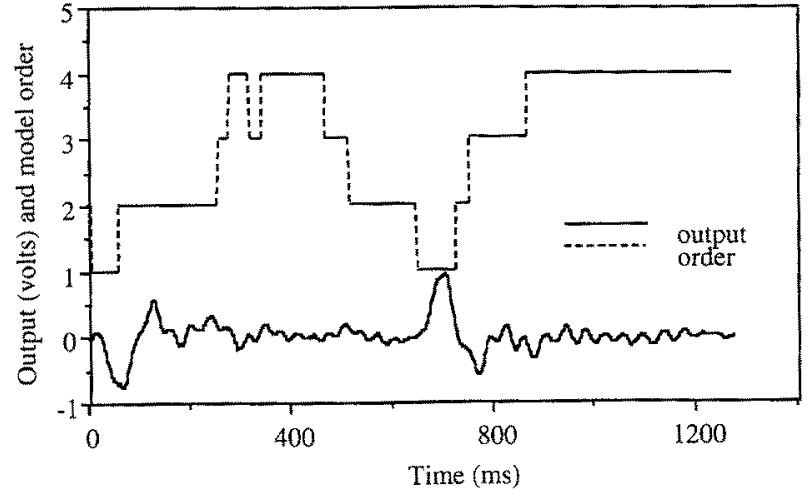

Fig. 5. The on-line order determination result at position $\left(0,0, Z_{\mathrm{xtax}}\right)$.

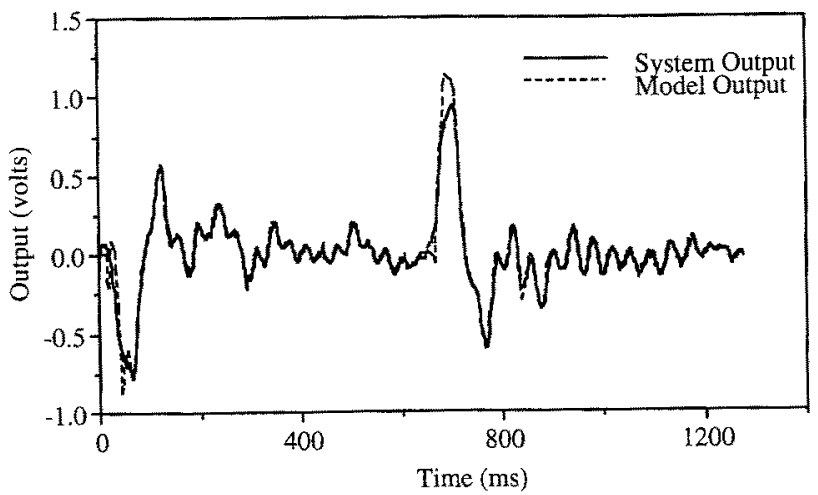

Fig. 6. The output two-step forecasting based on the on-line DDS at position $\left(0,0, Z_{\max }\right)$.

\section{Discussions:}

1. The on-line DDS approach can simultaneously identify the model order and parameters. The digital signal processor was able to complete both the coefficient estimation for the candidate model set $\operatorname{ARX}(n, n-1)(n=1,2, \ldots$, 8 ) and the on-line determination within a sampling interval of $2.5 \mathrm{~ms}$. The speed of the on-line DDS modelling makes it feasible for real-time applications.

2. A lower-order model, comparing the model obtained with the off-line modelling techniques, can be used for realtime applications using the on-line modelling technique. If the system is time varying, the on-line modelling technique provides a time-varying model, which can always track the most dominant modes at the current moment. However, an off-line model uses a single time invariant model to cover all the system responses in a batch of data. Thus, a high model order has to be used to capture all the vibration frequencies. However, these vibration modes may not occur simultaneously and a lower model order can be used in on-line modelling.

3. In the application, the length of the receding window will influence the on-line order determination results. A larger window length will lead to smoother order determination, but reduces the order tracking ability. Another important parameter in one-line order determination is the confidence level of the $F$-test.

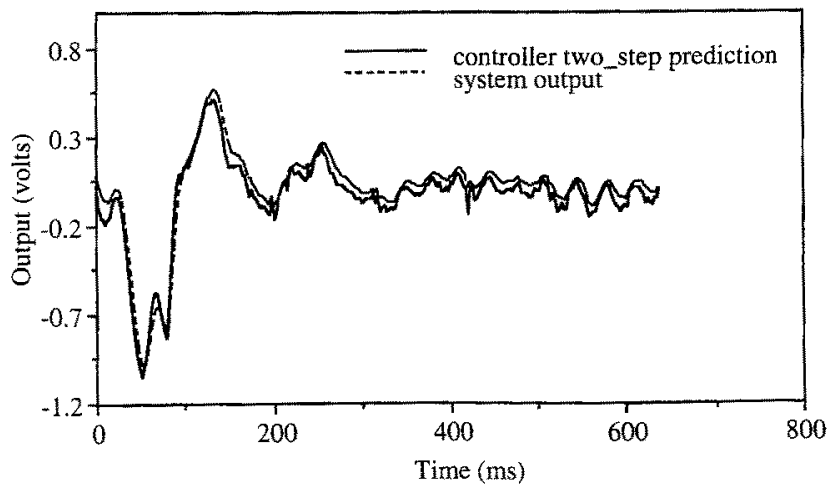

Fig. 7. The output two-step ahead forecasting based on the state estimates.

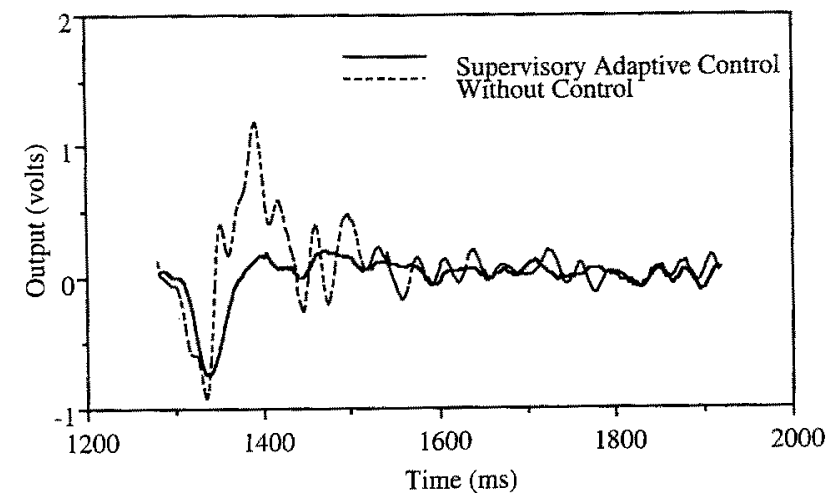

Fig. 8. The performance of the supervisory adaptive control.

\section{Supervisory Adaptive Control Experimental Results and Analysis}

The predictive control law (10) is obtained according to the system output prediction. Thus, the $i$-step ahead prediction performance is critical for the control performance. Figure 7 compares the two-step-ahead output prediction value, based on state estimates, with the real output.

The supervisory adaptive controller was used to control the CMM vibration. Figure 8 shows the experimental results. From the figure, we see that the controller reduces both the vibration magnitude and the vibration settling time. Figure 9

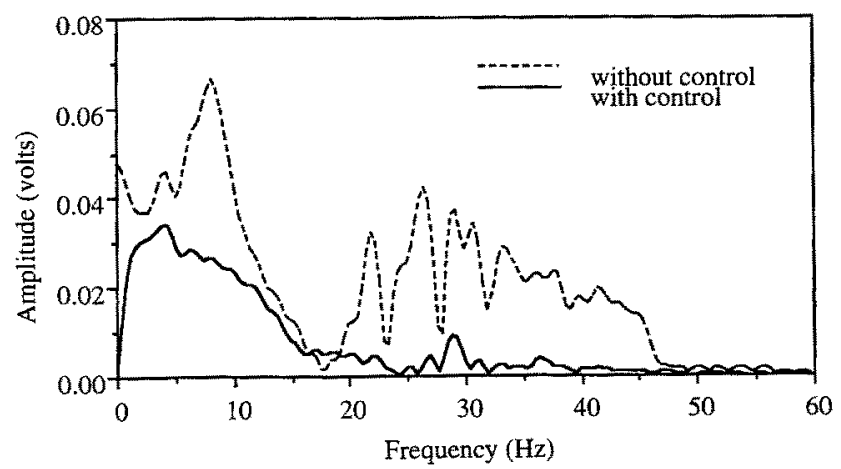

Fig. 9. The comparison of the output spectrum with and without control. 
Table 2. Summary of the experimental results using index (16)

\begin{tabular}{|c|c|c|c|c|}
\hline$J(s)$ status & start ( & stop $(+X)$ & start $(-X)$ & stop $(-X)$ \\
\hline Without control & 37.9 & 31.1 & 25.6 & 33.6 \\
\hline With control & 7.19 & 6.34 & 6.78 & 8.03 \\
\hline Improvement & $81.0 \%$ & $79.6 \%$ & $73.5 \%$ & $76.1 \%$ \\
\hline
\end{tabular}

shows a comparison of the output spectrum. A summary of the experimental results is shown in Table 2, where the control performance index $J(s)$ is defined as the sum of the squares of the vibration signal.

$$
J(s)=\sum_{k=1}^{256} y_{s}^{2}(k)
$$

where $s$ represents the control status: $y_{s}(k)$ is the vibration magnitude under the control status $s$.

\section{Discussions:}

1. Supervision of the model order determination is important in implementation. Very often model order switching results in improper system performance owing to the transient time required in the adaptive control scheme. A pre-set threshold $N_{f}$ is used to eliminate the unnecessary model order changes due to the statistical nature of the $F$ test. In fact, a controller generally has a robust boundary which can tolerate a certain amount of model mismatch. It is therefore recommended that for the on-line order determination, one should consider not only the results of the $F$-test (statistically if the model order changes or not), but also the robust boundary of the controller (if it is necessary to change the model order regarding the system stability concern). If the robust boundary can cover the mismatch of the model and system, then, the model order may be kept the same, even though the $F$-test indicates that the adequate model order should be changed. The approaches for the robust boundary determination can be found in [15] and [9].

2. In the application, the supervision of the noise mean is critical to the stability of the on-line system identification and adaptive control. The experiments have shown that the on-line modelling and state estimation will diverge if no supervision is applied.

3. Though the "detection of the identification condition" is generally important for system identification, it was removed in the design of the controller for this application because there was no identification condition failure observed throughout the experiments.

4. In the abrupt change detection, both the recursive $F$-test and CUSUM chart have proved to be very effective. The abrupt changes mostly occur when the CMM changes its direction of travel in the $X$-axis. The detection algorithm is finally modified to simplify the adaptive controller. Instead, information of changes in CMM motion $X$ direction, which is available in the CMM measurement program, is used as an indicator for the supervisory rules to detect occurrences of abrupt changes in the system.

\section{Conclusions}

A supervisory adaptive controller is designed for the structural vibration control of a coordinate-measuring machine. Experimental evaluation demonstrated that a $75 \%$ peak-to-peak reduction in the vibration magnitude and an $80 \%$ reduction in the vibration settling time have been achieved. Though the improvements are comparable with the results presented in $\mathrm{Lu}$ et al. [1], the experiments presented in this paper were conducted under normal CMM operating conditions, without tightening the belts to their maximum tension. Thus, the supervisory adaptive controller is more attractive in practical applications.

In the realisation of the controller, the forgetting factor selection is very important for the on-line modelling. A general rule is that a smaller forgetting factor (around 0.95) corresponds to a faster CMM speed, and vice versa. However, a trial and error approach was used in the experiments. It should be noticed that once the parameter is selected for a specific CMM speed, it can be applied for all the CMM movements in the working space under the speed. For a given $\mathrm{CMM}$, a database may be developed for the forgetting factors corresponding to different CMM movement speeds. Further study on the forgetting factor selection is desirable.

Though the supervisory adaptive controller was developed and tested for the CMM structural vibration control, the generic controller structure, algorithm, and TNS320C30 board implementation can be applied for many applications. As an example, the implementation and further development of the supervisory adaptive controller in a machining chatter prevention project is on-going, and the results will be presented in another paper.

\section{References}

1. E. Lu, J.Ni, Z. G.Huang and S. M. Wu, “An integrated lattice filter adaptive control system for time varying $\mathrm{CMM}$ structural vibration control," Proceedings of ASME 1992 winter meeting PED-Vol. 55, 1992.

2. K. J. Astrom and B. Wittenmark, Computer Controlled System: Theory and Design, Prentice Hall, 1990.

3. E. Irving, "Improving power network stability and unit stress with adaptive generator control", Automatica, 15, p. 31, 1979.

4. B. Egardt, Stability of Adaptive Controllers, Lecture Notes In Control and Information Sciences, Springer, Berlin, 1979.

5. P. E. Wellstead and S. P. Sacoff, "Extend self-turning algorithm", International Journal of Control, 34, p. 433, 1981.

6. T. R. Fortescue, L. S. Kershenbaum and B. E. Ydstie, "Implementation of self-turning regulators with variable forgetting factors", Automatica, 17, p. 831, 1981.

7. R. Schumann, K.-H. Lachmann and R. Isermann, "Towards applicability of parameter-adaptive control algorithms", Proceedings IFAC-Congress, Kyoto, Pergamon Press, Oxford, 1981.

8. R. Isermann and K.-H. Lachmann, "Parameter-adaptive control with configuration aids and supervision functions", Automatica, 21(6), pp. 625-638, 1985.

9. J. Shi, "On-line dynamic data system modeling and generalized forecasting compensation control with applications", $\mathrm{PhD}$ thesis, University of Michigan, 1992.

10. R. R. Bitmead, M. Gevers and V. Wertz, Adaptive Optimal Control: The Thinking Man's GPC, Prentice Hall, 1990.

11. K. J. Astrom, J. J. Anton and K.-E. Arzen, "Expert control", Automatica, 22(3), pp. 277-286, 1986. 
12. C. Richard Johnson, Lectures on Adaptive Parameter Estimation, Prentice Hall Advanced Reference Series, 1988.

13. N. Basseville and A. Benveniste, Detection of Abrupt Changes in Signals and Dynamical Systems, Springer-Verlag, 1986.

14. D. C. Montgomery, Introduction to Statistical Quality Control, 2nd edn, Wiley, NY, 1991.

15. E. Yaz and Xiaoru Niu, "Stability robustness of linear discretetime systems in the presence of uncertainty", Journal of Control, 50(1), pp. 173-182, 1989.

\section{Appendix A. Model Information Matrix and $J_{K}(t)$}

A model information matrix is defined using the system input and output information. To define the model information matrix for a piecewise stationary process, let us define the following vectors

$$
\begin{aligned}
\mathbf{y}_{k}(t)= & {[\beta(t, n) y(k), \beta(t, n+1) y(k+1), \ldots,} \\
& \beta(t, t-1) y(t-n+k-1), \beta(t, t) y(t-n+k)]_{(t-n+1)}^{T}
\end{aligned}
$$

$$
\begin{aligned}
\mathbf{u}_{k}(t)= & {[\beta(t, n) u(k), \beta(t, n+1) u(k+1), \ldots,} \\
& \beta(t, t-1) u(t-n+k-1), \beta(t, t) u(t-n+k)]_{(t-n+1)}^{T}
\end{aligned}
$$

where $t(t \geq n)$ is the time index, $k=0,1,2, \ldots, n$, and $\beta(t, i)$ $(n \leq i \leq t)$ are the weighting coefficients. Also define

$$
\mathbf{X}_{k}(t)=\left[y_{0}(t), \mathbf{u}_{0}(t), \ldots, \mathbf{y}_{k-1}(t), \mathbf{u}_{k-1}(t), \mathbf{y}_{k}(t)\right]_{(t-n+1)} \times(2 k+1)
$$

Then, the model information matrix, $\mathbf{M}(t)$, is defined as

$$
\mathbf{M}(t)=\left[\mathbf{X}_{n}^{T}(t) \mathbf{X}_{n}(t)\right]_{(2 n+1) \times(2 n+1)}^{-1}
$$

The $\mathbf{J}_{k}(t)$ used in the $\mathbf{D}$ matrix (see equation (3)) is expressed as:

$$
J_{k}(t)=\left(y_{k}(t)-\mathbf{H}_{k}(t) \hat{\theta}_{k}(t)\right)^{x}\left(\mathbf{y}_{k}(t)-\mathbf{H}_{k}(t) \hat{\theta}_{k}(t)\right)
$$

where $J_{0}(t)=\mathbf{y}_{0}^{T}(t) \mathbf{y}_{0}(t)$ and $\mathbf{H}_{k}(t)$ is defined as

$$
\mathbf{H}_{k}(t)=\left[\mathbf{X}_{k-1}(t), \mathbf{u}_{k-1}(t)\right]
$$

